\title{
Correction to: Differential effects of sevoflurane on the growth and apoptosis of human cancer cell lines
}

\author{
Takahiro Hirai $^{1} \cdot$ Yuko Konishi $^{2} \odot \cdot$ Shoko Mizuno $^{3} \cdot$ Zhou Rui $^{1} \cdot$ Yao Sun ${ }^{1} \cdot$ Kimitoshi Nishiwaki $^{1}$
}

Published online: 3 December 2019

C Japanese Society of Anesthesiologists 2019

\section{Correction to: Journal of Anesthesia \\ https://doi.org/10.1007/s00540-019-02701-w}

The article Differential effects of sevoflurane on the growth and apoptosis of human cancer cell lines, written by Takahiro Hirai, Yuko Konishi, Shoko Mizuno, Zhou Rui, Yao Sun and Kimitoshi Nishiwaki, was originally published electronically on the publisher's internet portal (currently SpringerLink) on 31 October 2019 with open access. With the author(s)' decision to step back from Open Choice, the copyright of the article changed on 5 December 2019 to $($ ) Japanese Society of Anesthesiologists 2019 and the article is forthwith distributed under the terms of copyright.

The original article was updated.

The original article can be found online at https://doi.org/10.1007/ s00540-019-02701-w.

\section{Yuko Konishi}

ykonishi@med.nagoya-u.ac.jp

1 Department of Anesthesiology, Nagoya University Graduate School of Medicine, 65 Tsurumai-cho, Showa-ku, Nagoya, Aichi 466-8550, Japan

2 Endowed Division of Perioperative Management, Nagoya University Graduate School of Medicine, 65 Tsurumai-cho, Showa-ku, Nagoya, Aichi 466-8550, Japan

3 Aichi Spine Institute, 31-1, Kamiike, Goromaru, Inuyama, Aichi 484-0066, Japan
Publisher's Note Springer Nature remains neutral with regard to jurisdictional claims in published maps and institutional affiliations. 